\title{
Características tecnológicas de la madera de dos especies de Costa Grande, Guerrero, México
}

\section{Technological characteristics of two wood species from Costa Grande, Guerrero, México}

\author{
Elizandro Pineda-Herrera1, Carmen de la Paz Pérez-Olvera2, \\ Raymundo Dávalos-Sotelo 3 y Juan Ignacio Valdez-Hernández 4
}

\begin{abstract}
RESUMEN
Se describen las características anatómicas, físicas, mecánicas y tiempo de secado al aire libre de la madera de Enterolobium cyclocarpum (Jacq.) Griseb. y Hura polyandra Baill., recolectadas en una selva mediana subcaducifolia de Zihuatanejo de Azueta, Guerrero, México. Para la descripción anatómica se utilizaron tablillas de $15 \mathrm{~cm} \times 7 \mathrm{~cm} \times 1 \mathrm{~cm}$ y preparaciones fijas de cortes de $20 \mathrm{~mm}$ de grosor, ambas en secciones transversales, tangenciales y radiales y preparaciones de material disociado. Para los ensayos físicos y mecánicos se emplearon probetas libres de defectos de diferentes dimensiones a un contenido de humedad de $12 \%$. De cada característica se hizo un análisis estadístico y se clasificaron de acuerdo con la media. Para el secado al aire libre se acomodaron tablones y prismas en una pila. Las especies presentan vasos de diámetro mediano, radios uniseriados, extremadamente bajos y fibras de longitud mediana de diámetro fino. Presentan diferencias en el número de poros y la longitud de los vasos, en el número y anchura de los radios, en el grosor de la pared de las fibras y en la anchura de los anillos de crecimiento. En las propiedades físicas, difieren en la densidad, en su coeficiente de anisotropía y en el punto de saturación de la fibra. Las contracciones son bajas, excepto la radial de E. cyclocarpum que es muy baja; esta especie es poco estable y $H$. polyandra es muy estable. Los valores de las propiedades mecánicas y el tiempo de secado son diferentes entre ambas especies.
\end{abstract}

PALABRAS CLAVE:

Enterolobium cyclocarpum, Hura polyandra, selva mediana subcaducifolia, madera secada.

\begin{abstract}
The anatomical, physical, and mechanical features, and drying time of wood from Enterolobium cyclocarpum (Jacq.) Griseb. (Parota, Mimosoideae, Leguminosae) and Hura polyandra Baill. (Habillo, Euphorbioideae, Euphorbiaceae) collected in a semideciduous forest of Zihuatanejo de Azueta, Guerrero, Mexico, are described. For the anatomical description, pieces of $15 \mathrm{~cm} \times 7 \mathrm{~cm} \times 1 \mathrm{~cm}$ and slides $20 \mathrm{~mm}$-thick, in transverse cross-section, tangential and radial directions and preparations of dissociated material were used. For the physical and mechanical tests small clear specimens of various dimensions, conditioned to a moisture content of $12 \%$, were utilized. For each feature measured, univariate statistical analyses were performed and results were classified according to the mean. For air drying time tests, boards and prisms were placed in a stack with spacers between them. The species have medium diameter vessels, extremely low uniseriate rays, and medium length fibers
\end{abstract}


of fine diameter. They differ in the number of pores and the length of the vessels, the number and width of rays, on the wall thickness of fibers and the width of the growth rings. For the physical properties, shrinkage values are low, except the radial shrinkage for $E$. cyclocarpum, which is very low. This species is dimensionally not stable whereas $H$. polyandra is highly stable. There are significant differences between species in the mechanical valules and the drying time.

KEYWORDS:

Enterolobium cyclocarpum, Hura polyandra, deciduous tropical forest, air dried wood.

\section{INTRODUCCIÓN}

Las selvas secas en México cubren alrededor de $11,26 \%$ del territorio nacional, dividiéndose en: selvas bajas caducifolias, selvas bajas subcaducifolias, selvas medianas caducifolias y selvas medianas subcaducifolias (INEGI, 2005). Se distribuyen desde Sonora hasta Chiapas, en la Vertiente del Golfo en Tamaulipas, San Luis Potosí, porción central de Veracruz y norte de la Península de Yucatán. Están ampliamente representadas en la Cuenca del Balsas (Pennington y Sarukhán, 2005), de forma general se caracterizan por presentar un periodo de secas marcado de noviembre a mayo.

La región de Guerrero, denominada Costa Grande, es la de mayor extensión estatal $\left(14711 \mathrm{~km}^{2}\right)$ y está conformada por siete municipios: Atoyac de Álvarez, Coahuayutla de José María Izazaga, Coyuca de Benítez, La Unión de Isidoro Montes de Oca, Tecpan de Galeana, Petatlán y Zihuatanejo de Azueta, en la cual se encuentran altitudinalmente selvas bajas caducifolias (0 m a $380 \mathrm{~m})$ y selvas medianas subcaducifolias $(380 \mathrm{~m}$ a 650 m) (Pineda et al., 2012). Entre las especies arbóreas representativas de la región están: Enterolobium cyclocarpum (parota) y Hura polyandra (habillo).
Rodríguez (2009) menciona que en el municipio de La Unión de Isidoro Montes de Oca, E. cyclocarpum tiene una participación de $16 \%$ en la industria del mueble, pero la madera se obtiene de forma clandestina. Para $H$. polyandra no se conocen usos regionales. Morán et al. (2008), en su estudio de algunos ejidos de Costa Grande, resaltan la necesidad de generar información que apoye a los tomadores de decisiones del sector forestal, así como difundir las ventajas socioambientales de manejar las selvas y los bosques de la región.

La madera de parota en otros estados es valorada para: artesanías, canoas, carpintería, carretas, construcciones rurales, chapa, decoración de interiores, duela, ebanistería, embarcaciones, lambrín, madera aserrada, muebles, paneles, postes, ruedas de carretas, triplay y utensilios de cocina (Robles, 1978; Guridi, 1980; Pennington y Sarukhán, 2005; Silva, 2008; Román et al., 2011).

La madera de habillo es valorada para artesanías, carpintería, construcciones rurales, chapas, embalajes, mangos para herramientas, muebles y triplay (Pennington y Sarukhán, 2005; Silva, 2008; Román et al., 2011).

Estos usos están sustentados en estudios anatómicos, físicos y mecánicos de la madera de ambas especies. Enterolobium cyclocarpum se ha estudiado en ejemplares de selvas húmedas de la vertiente del Golfo por Cárdenas (1971); Robles (1978), Corral (1985) y González et al. (2004). De selvas secas de la vertiente del Pacífico se conoce la investigación de Guridi (1980), quien describe las características macroscópicas de la madera.

Zavala (2000), en su estudio sobre especies tropicales, presenta el programa 
de secado de E. cyclocarpum, considerándola con buenos resultados. Silva (2008) la considera moderadamente difícil de secar al aire libre ya que presenta deformaciones importantes; el mismo autor indica que Hura polyandra es difícil de secar, tanto al aire libre como en estufa. La importancia del secado es que permite que la humedad de la madera esté en equilibrio con la humedad del ambiente, para que no haya problemas durante su servicio.

\section{OBJETIVO}

Determinar las características anatómicas, físicas, mecánicas y tiempo de secado al aire libre de la madera de Enterolobium cyclocarpum (parota) y Hura polyandra (habillo) de una selva mediana subcaducifolia de Costa Grande, Guerrero.

\section{METODOLOGÍA}

El material de estudio se recolectó en una selva mediana subcaducifolia de la localidad Los Varillos (17 $53^{\circ}$ '58' N, $101^{\circ} 26^{\prime}$ 68 " O), perteneciente al ejido La Laja y anexos, del municipio de Zihuatanejo de Azueta, Costa Grande, Guerrero, a una altura de 440 msnm (Fig. 1). El clima es cálido subhúmedo con lluvias en verano (Aw1) (INEGI, 2005) con una precipitación anual entre $1100 \mathrm{~mm}$ y $1500 \mathrm{~mm}$ y temperatura promedio de $28{ }^{\circ} \mathrm{C}$ (SMN, 2012). Con respecto a la geomorfología de Los Varillos, se trata de una meseta rodeada por laderas con pendientes pronunciadas y lomeríos moderados. Las rocas son ígneas extrusivas y metamórficas del Cenozoico superior. Los suelos, de origen volcánico, son: Cambisoles, Luvisoles, Leptosoles y Regosoles (INEGI, 2009).

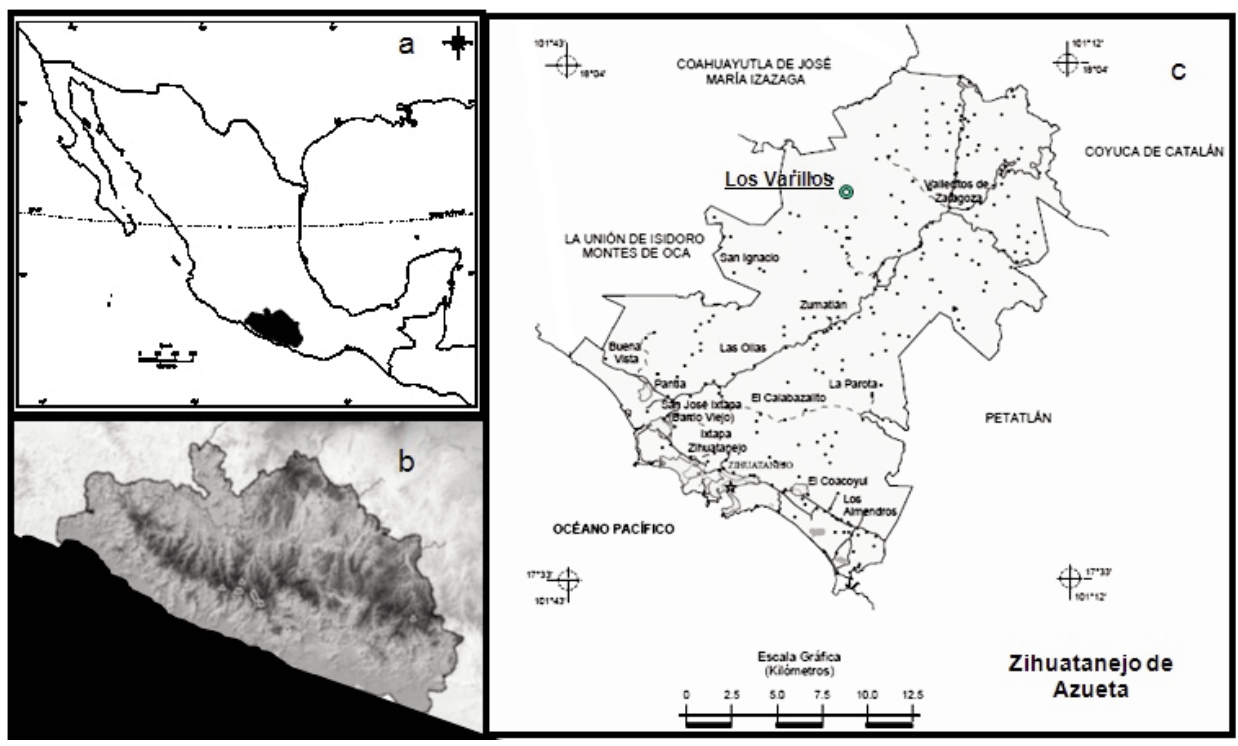

Figura 1. Zona de estudio a. México. b. Estado de Guerrero. c. Los Varillos. 
Estas selvas se caracterizan porque la temporada seca puede durar de 7 a 8 meses; fisonómicamente la altura de los árboles va de $7 \mathrm{~m}$ a $25 \mathrm{~m}$ y en promedio es de $15 \mathrm{~m}$. Algunas de las especies encontradas son: Enterolobium cyclocarpum (Jacq.) Griseb, Hura polyandra Baill., Tabebuia rosea (Bertol.) DC., Licania arborea Seem. y Bursera instabilis McVaugh \& Rzed.

Se recolectaron dos árboles por especie con una altura total aproximada de $8 \mathrm{~m}$ y de $30 \mathrm{~cm}$ a $35 \mathrm{~cm}$ de diámetro cada uno. De cada árbol se obtuvieron cuatro trozas de base a copa, la primera de 1,30 m se utilizó para el estudio anatómico y las otras tres de $1,20 \mathrm{~m}$ para los ensayos físicos, mecánicos y de secado (Fig. 2).
De las trozas para anatomía se obtuvieron tablillas transversales, tangenciales y radiales de $15 \mathrm{~cm} \times 7 \mathrm{~cm} \times 1 \mathrm{~cm}$ para describir las características macroscópicas de acuerdo con Tortorelli (1956), para el color se utilizaron las tablas de Munsell (1990). Las tablillas transversales se obtuvieron de cuatro rodajas de $1 \mathrm{~cm}$ de grosor cortadas a la altura de 1,26 m. Para el estudio microscópico se cortaron dos rodajas a la altura de $1,30 \mathrm{~m}$, de las que se obtuvieron cubos que se ablandaron a ebullición en agua destilada por 24 h para E. cyclocarpum y 42 hrs para $H$. polyandra. De los cubos se obtuvieron cortes transversales, tangenciales y radiales de $20 \mu \mathrm{m}$ de grosor en un microtomo de deslizamiento en el Laboratorio de Anatomía de la Madera de la Universidad Autónoma Metropolitana unidad Iztapalapa. a
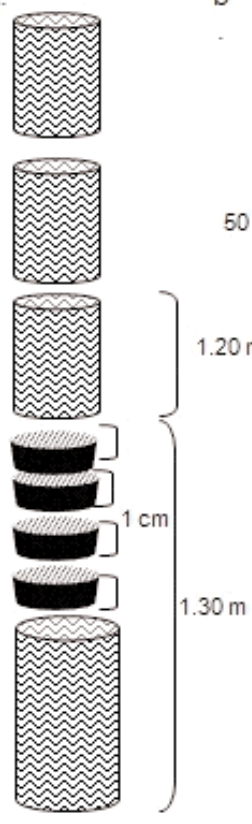

b
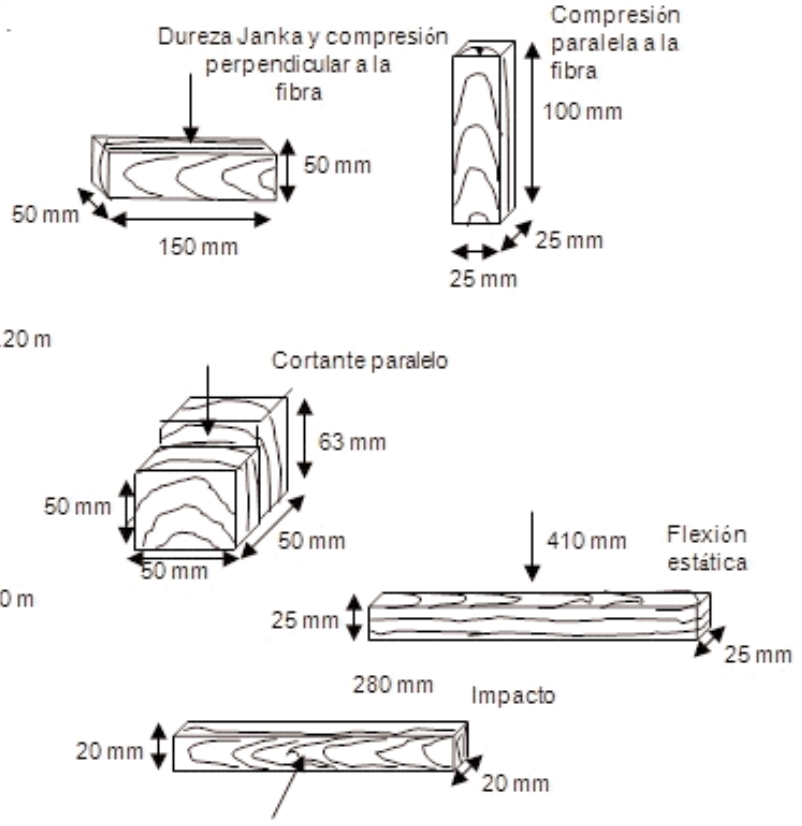

Figura 2. Material de estudio. a. Trozas y rodajas para anatomía, pruebas físicas, mecánicas y tiempo de secado. $b$. Probetas para ensayos mecánicos. 
Los cortes se tiñeron con fucsina básica al 0,05\% durante 30 segundos y después en azul astra al 0,5\% durante dos minutos. Se deshidrataron en una serie gradual de alcoholes del $50 \%, 70 \%$, $85 \%, 96 \%$ y $100 \%$. Después de deshidratados se les puso una gota de aceite de clavo por dos minutos para mayor contraste, posteriormente se colocaron en una mezcla de clavo con xilol, se pasaron por xilol y se montaron con resina sintética (Sandoval, 2005).

De los mismos cubos se cortaron pequeñas astillas para obtener material disociado con una mezcla de ácido acético, ácido láctico, ácido nítrico y glicerina, se tiñeron con Pardo de Bismarck y se montaron con gelatina glicerinada de acuerdo a Johansen (1940). Se realizaron 25 mediciones por carácter y por árbol, los que se sometieron a un análisis estadístico y se clasificaron con base en la media de acuerdo con Chattaway (1932) y con IAWA Committee (1937, 1939, 1989) (tablas 1 y 2). Los elementos no mensurables se describieron con base en
IAWA (1964) y los cristales de acuerdo con Chattaway (1955).

Las trozas para el material destinado a ensayos físicos y mecánicos, se trasladaron a las instalaciones del Instituto de Ecología, A.C. en Xalapa, Ver. De dichas trozas se cortaron tablones tangenciales $y$ radiales de 0,03 $\mathrm{m} \times 0,12 \mathrm{~m} \times 1,20 \mathrm{~m}$ y prismas de $0,06 \mathrm{~m} \times 0,06 \mathrm{~m} \times 1,20 \mathrm{~m}$, para medir el tiempo de secado y posteriormente elaborar los especímenes para los ensayos físicos y mecánicos. Para el secado al aire libre, a un contenido de humedad de $16 \%$, que es el promedio del obtenido en las condiciones de humedad relativa y temperatura del ambiente en la región centro del Golfo de México; para el periodo de elaboración de las probetas se acomodaron los tablones y los prismas en una pila elevada sobre el piso de $1,35 \mathrm{~m}$ de largo x 1,20 m de ancho x 1,50 m de altura, con separadores entre las capas de madera permitiendo la circulación libre del aire en torno a cada una y se cubrieron para evitar que estuvieran directamente expuestas al sol y a la lluvia (Fig. 3).

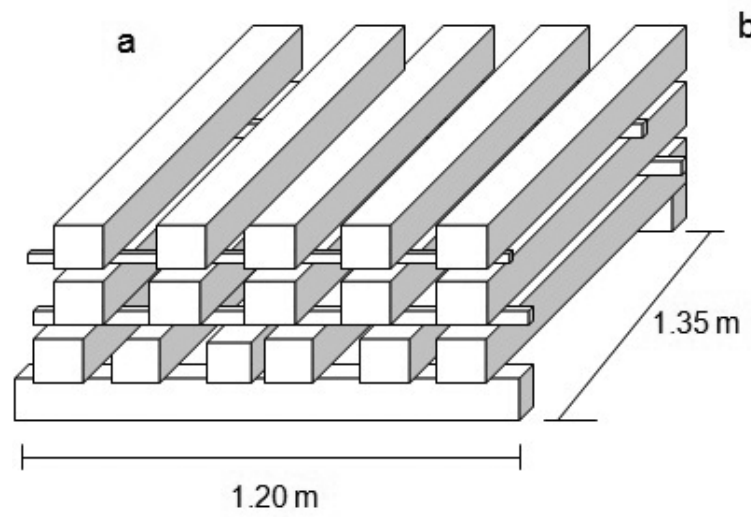

b

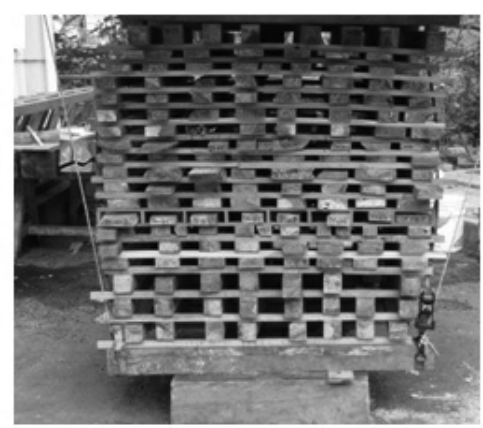

Figura 3. Arreglo de prismas y tablas en la pila de secado al aire libre. a. Dimensiones de la pila en el plano horizontal. $b$. Vista frontal. 


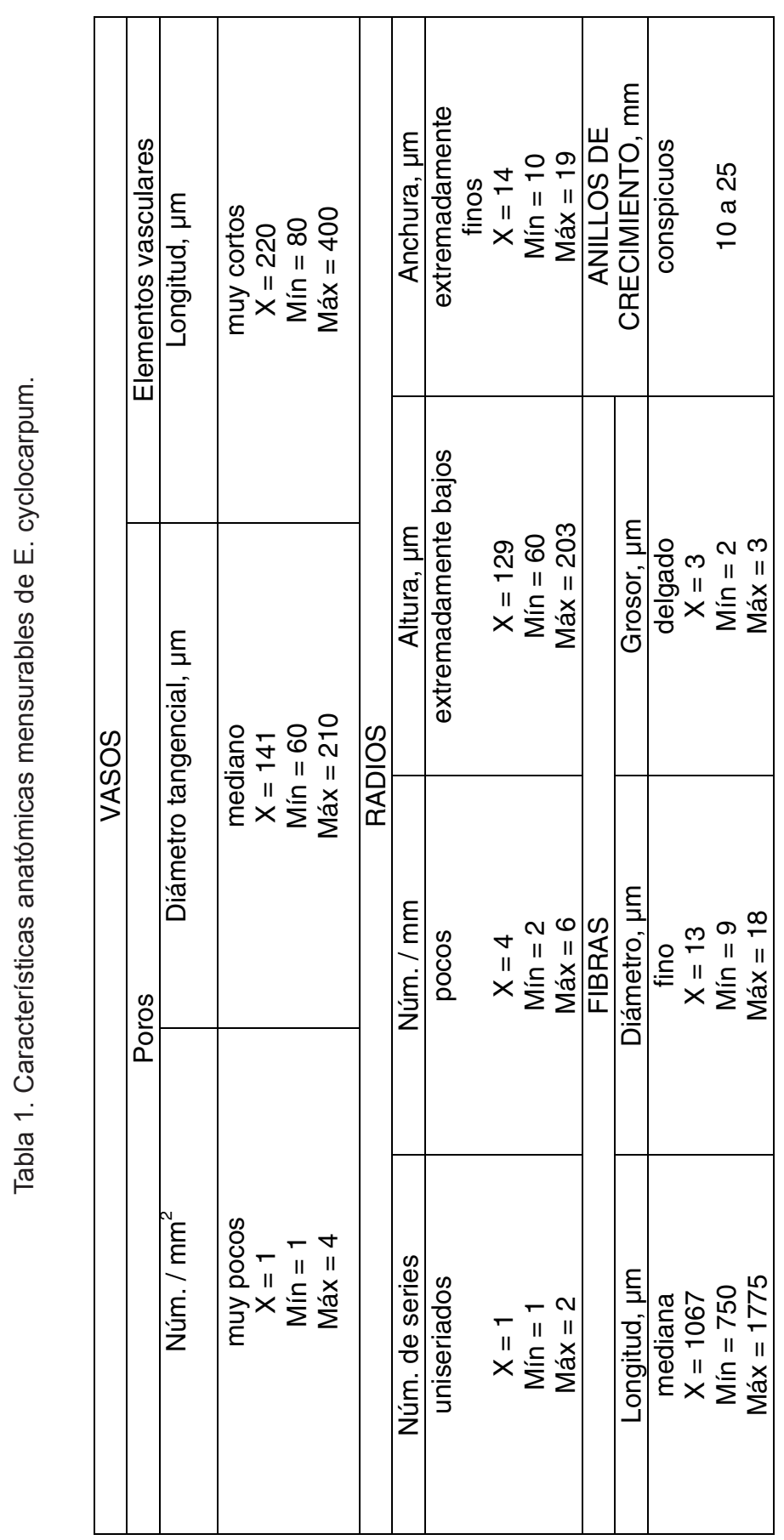




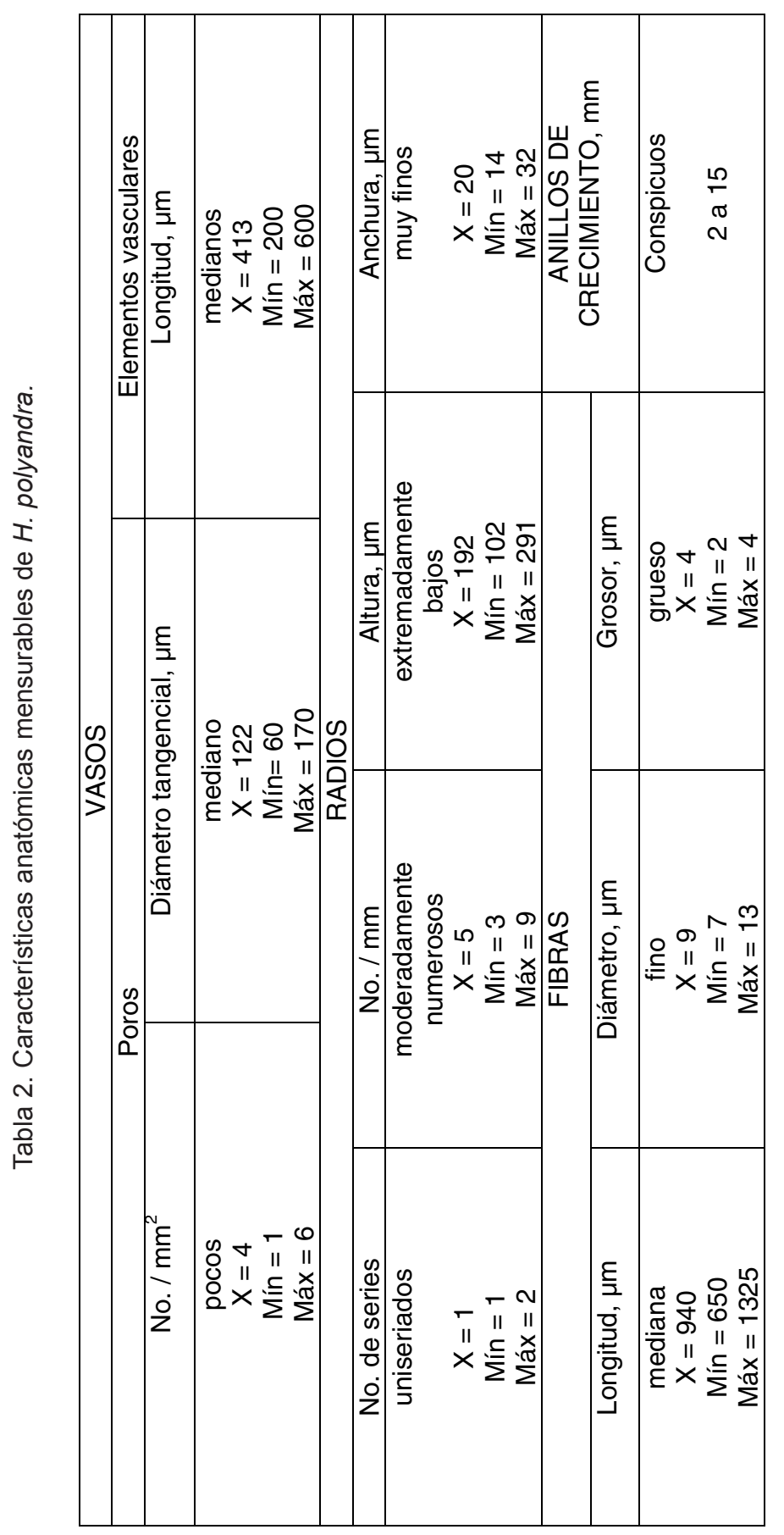


De los tablones se obtuvieron las probetas para los ensayos físicos y de los prismas las probetas para determinar las propiedades mecánicas, basados en la norma ASTM (2005) (Fig. 2). Después del proceso de secado al aire libre, las tablas y prismas se acondicionaron en una cámara con condiciones controladas hasta alcanzar un contenido de humedad cercano al $12 \%$. De cada probeta se obtuvieron muestras para la determinación de la densidad básica.

A cada propiedad se le hizo un análisis estadístico univariado, las propie- dades físicas se clasificaron de acuerdo con la media con base en EcheniqueManrique et al. (1975) y Sallenave (1955) para el punto de saturación de la fibra; las propiedades mecánicas de acuerdo con Dávalos y Bárcenas (1999) (tablas 3 y 4). Las pruebas se hicieron en el Laboratorio de Investigación y Certificación de Productos Maderables del Instituto de Ecología, A.C., en una máquina universal de $250 \mathrm{kN}$ de capacidad. Las pruebas de impacto se realizaron en una máquina para ensayos de tenacidad modelo FPL. La clasificación para tiempo de secado se desarrolló específicamente para este

Tabla 3. Características físicas de E. cyclocarpum y H. polyandra.

\begin{tabular}{lcccc}
\hline & E. cyclocarpum & \multicolumn{2}{c}{ H. polyandra } \\
\hline Densidad básica, PA/VV & Muy liviana & 0,29 & Moderadamente & 0,48 \\
& & & pesada \\
Contracción tangencial, \% & Baja & 4,63 & Baja & 4,89 \\
Contracción radial, \% & Muy baja & 1,83 & Baja & 2,97 \\
Contracción volumétrica & Muy baja & 6,46 & Baja & 7,86 \\
Coeficiente de anisotropía & Poco estable & 2,52 & Muy estable & 1,65 \\
Punto de saturación de la fibra, \% & Medio & 33,4 & Bajo & 21,4 \\
\hline
\end{tabular}

Tabla 4. Características mecánicas de E. cyclocarpum y H. polyandra. *

\begin{tabular}{lcccc}
\hline Propiedad mecánica & E. cyclocarpum & H. polyandra \\
\hline Dureza Janka lateral, kN & Suave & 2,28 & Muy dura & 4,40 \\
Dureza Janka longitudinal, kN & Suave & 3,04 & Muy dura & 6,17 \\
Módulo de ruptura en flexión (MOR), MPa & Flexible & 41,4 & Resistente & 76,8 \\
Módulo de elasticidad en flexión (MOE), GPa & Muy flexible & 3,83 & Moderadamente flexible & 8,08 \\
Esfuerzo máximo en compresión paralela, MPa & Poco resistente & 26,1 & Resistente & 44,1 \\
Módulo de elasticidad en compresión & & & & \\
paralela (E), GPa & Muy flexible & 3,93 & Moderadamente flexible & 8,70 \\
Esfuerzo en el límite de proporcionalidad & & & Resistente & 7,21 \\
en compresión perpendicular, MPa & Poco resistente & 3,47 & Muy resistente & 11,6 \\
Esfuerzo máximo en cortante paralela,MPa & Moderadamente resistente & 7,11 & Poco resiliente & 0,264 \\
Impacto (Tenacidad), J/m3 & Poco resiliente & 0,222 & \\
\hline
\end{tabular}

*Valores ajustados al 12\% de Contenido de humedad (ASTM, 1998) 
trabajo, a partir de los datos registrados de variación diaria de contenido de humedad en proporción al contenido de humedad en equilibrio para el periodo en el que se hicieron los ensayos.

Las características botánicas, distribución geográfica y nombres comunes se consultaron en Pennington y Sarukhán (2005), a excepción de las medidas dasométricas y la distribución altitudinal que se obtuvieron directamente en la zona de estudio (Pineda et al., 2012). Las muestras para anatomía se depositaron en la Xiloteca UAMIZ y los ejemplares botánicos se registraron en el Herbario Metropolitano UAMIZ Dr. Ramón Riba y Nava (Tabla 5).

\section{RESULTADOS}

\section{Características botánicas, anatómicas, físicas, mecánicas y tiempo de secado de la madera}

Enterolobium cyclocarpum (Jacq.)

Griseb. (Lámina 1, figs. a-f)

\section{Características botánicas}

Familia: Leguminosae Subfamilia: Mimosoideae.

Nombres comunes: Parota, guanacastle, orejón.
Distribución geográfica: En la vertiente del Pacífico, desde Sinaloa hasta la depresión Central de Chiapas; incluyendo la Cuenca del Balsas. En la vertiente del Golfo, desde el Sur de Tamaulipas hasta el Norte de Yucatán.

Distribución altitudinal: 0 m a 800 msnm.

Árbol hermafrodita, de $7 \mathrm{~m}$ a $25 \mathrm{~m}$ de altura y diámetros de $0,3 \mathrm{~m}$ a $1 \mathrm{~m}$ (DAP), corteza fisurada de color castaño rojizo (HUE 2.5YR 4/6), con numerosas lenticelas. Hojas compuestas, de hasta $40 \mathrm{~cm}$ de largo, bippinadas, con numerosos foliolos de $10 \mathrm{~mm}$ a $12 \mathrm{~mm}$ de largo. Flores en cabezuelas, blancas, $1,5 \mathrm{~cm}$ a $2 \mathrm{~cm}$ de diámetro. Fruto en vaina lignificada, en forma de media luna, de $7 \mathrm{~cm}$ a $12 \mathrm{~cm}$ de diámetro. Semillas ovoides y planas, de $1,5 \mathrm{~cm}$ a $2,3 \mathrm{~cm}$ de diámetro.

\section{Características anatómicas}

Macroscópicas (Lámina 1, figs. g-i)

La madera presenta diferencia de color entre albura y duramen, la albura es blanca (HUE 2.5Y8/2) y el duramen es castaño claro (HUE 7.5YR 6/4), sin olor ni sabor característicos, brillo mediano, veteado suave, textura fina e hilo oblicuo. Anillos de crecimiento conspicuos.

Tabla 5. Registro de las especies

\begin{tabular}{lcccc}
\hline Especie & Familia & Nombres comunes & \multicolumn{2}{c}{ Registros UAMIZ } \\
& subfamilia & & Xiloteca & Herbario \\
\hline \multirow{3}{*}{ E. cyclocarpum } & Leguminosae & Parota, guanacastle, & M-280 & M-281 \\
& Mimosoideae & orejón & 73423 & 73424 \\
H. polyandra & Euphorbiaceae & Habillo, jabilla & M-282 & M-283 \\
& Euphorbioideae & & 73427 & 73428 \\
& & & & \\
\hline
\end{tabular}




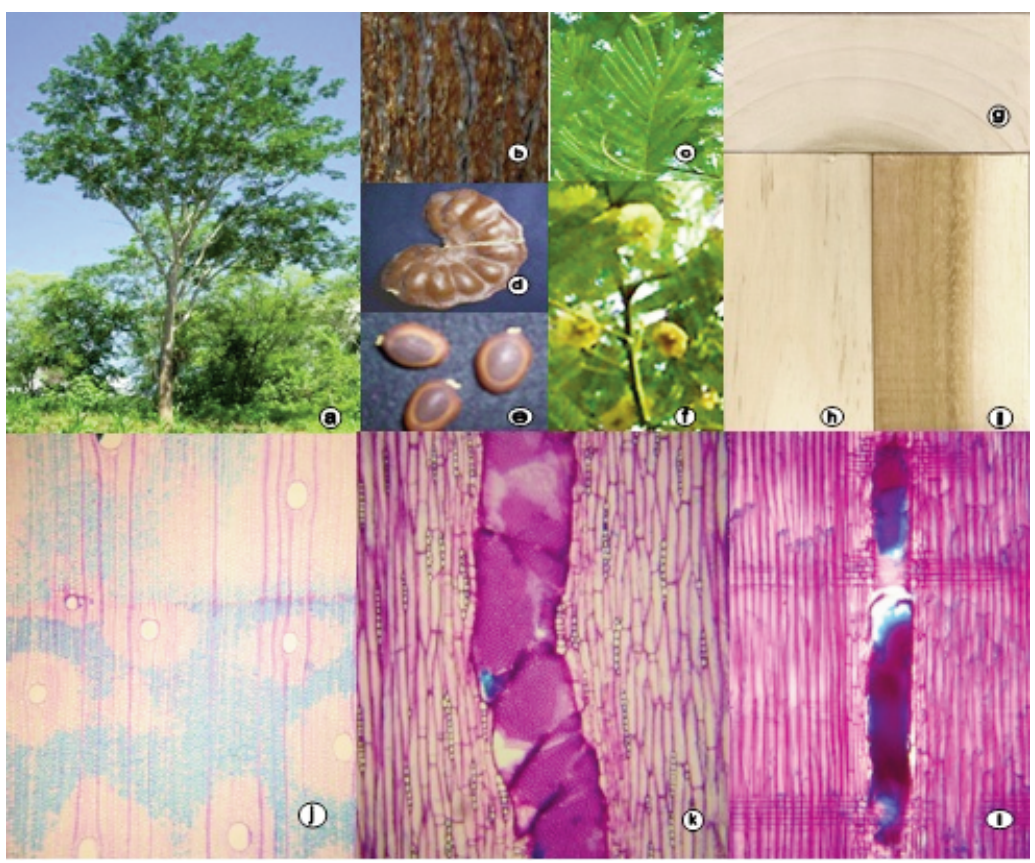

Lámina 1. Enterolobium cyclocarpum. a. árbol. b. corteza. c. hoja. d. fruto. e. semillas. $f$. flor. Tablillas: $g$. transversal. $h$. tangencial. $i$. radial. Cortes: $j$. transversal $(5 x)$. k. tangencial (10x). I. radial (10x).

Microscópicas (Lámina 1, figs. j-l; Lámina 3, figs. a-c; Tabla 1)

La madera es de porosidad difusa, los poros son muy pocos, de diámetro tangencial mediano, la mayoría solitarios, algunos múltiples de 2 a 4 . Los elementos vasculares son muy cortos, sus paredes tienen puntuaciones areoladas alternas, ovales, ornamentadas y platina de perforación simple. Algunos presentan gomas. El parénquima axial es paratraqueal vasicéntrico, de 6 a 15 células de anchura. Algunas células parenquimáticas presentan cristales prismáticos dentro de cámaras. Los radios son homogéneos, uniseriados, pocos, extremadamente bajos y extremadamente finos. Las fibras son de longitud mediana, diámetro fino y pared delgada. Los anillos de crecimiento miden desde $10 \mathrm{~mm}$ hasta $25 \mathrm{~mm}$ de ancho, marcados por 2 a 4 hileras de fibras.

\section{Características físicas (Tabla 3)}

La madera es muy liviana, de contracción tangencial y volumétrica bajas y contracción radial muy baja, poco estable y salida de agua libre rápida.

\section{Características mecánicas (Tabla 4)}

La madera es suave, flexible, poco resistente a la compresión, moderadamente resistente al cortante y poco tenaz. 
Características de secado al aire libre (Tabla 6)

Los resultados de la prueba de secado al aire libre para las dos especies estudiadas se muestran en la figura 4.

La madera es de secado rápido (tiempo de secado 25 días para alcanzar el CHE).
Hura polyandra Baill. (Lámina 2,

figs. a-g)

\section{Características botánicas}

Familia: Euphorbiaceae. Subfamilia: Euphorbioideae.

Nombres comunes: Habillo, jabilla, haba de San Ignacio.

Tabla 6. Características de tiempo de secado de E. Cyclocarpum y H. polyandra.

\begin{tabular}{lcc}
\hline & E. cyclocarpum & H. polyandra \\
\hline Contenido de humedad inicial, \% & 154 & 70 \\
Contenido de humedad final, \% & 16 & 16 \\
Tiempo de secado, días & 25 (rápido) & 35 (lento) \\
\hline
\end{tabular}

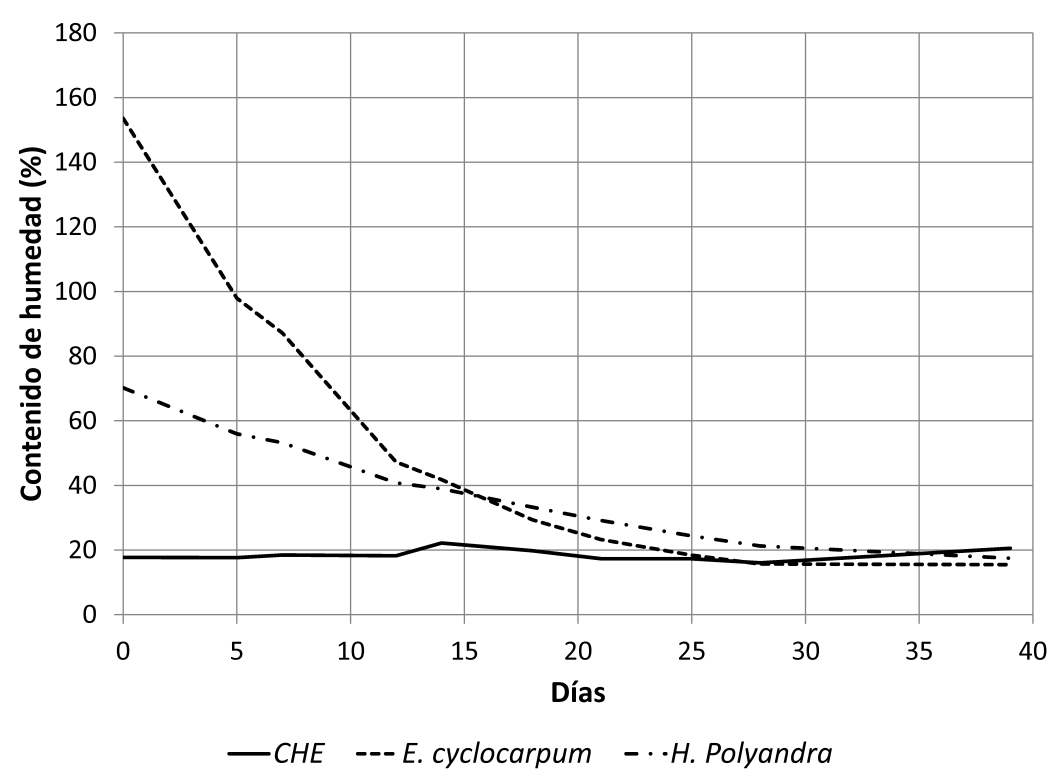

Figura 4. Curvas de secado para especies estudiadas 
Distribución geográfica: En la vertiente del Pacífico, desde Sinaloa hasta la depresión central y norte de Chiapas, incluyendo la Cuenca del Balsas. En la vertiente del Golfo desde el sureste de San Luis Potosí hasta el norte de Quintana Roo.

Distribución altitudinal: 150 m a 1000 msnm

Árbol monoico, de $7 \mathrm{~m}$ a $25 \mathrm{~m}$ de altura y diámetros de 0,30 m a $1 \mathrm{~m}$ (DAP), corteza lisa de color castaño claro (HUE 7.5 YR $6 / 3$ ) con numerosas espinas. Hojas simples, de hasta $17 \mathrm{~cm}$ de largo, ovadas a orbiculares. Flores masculinas en espiga de $12 \mathrm{~cm}$ a $16 \mathrm{~cm}$ de largo, las femeninas solitarias en pedúnculos de
$6 \mathrm{~cm}$ a $7 \mathrm{~cm}$ de largo. Fruto en cápsula multivalvada y lignificada de $5 \mathrm{~cm} \times 10 \mathrm{~cm}$ de diámetro, cubierta con abundantes lenticelas. Semillas circulares y planas, de $3 \mathrm{~cm}$ de diámetro.

\section{Características anatómicas}

Macroscópicas (Lámina 2, figs. $h$-j) La madera presenta diferencia de color entre albura y duramen, la albura es blanca (HUE 2.5Y 8/2) y el duramen es castaño claro (HUE7.5YR 6/3), sin olor ni sabor característicos, brillo mediano, veteado suave, textura fina e hilo recto. Anillos de crecimiento conspicuos.

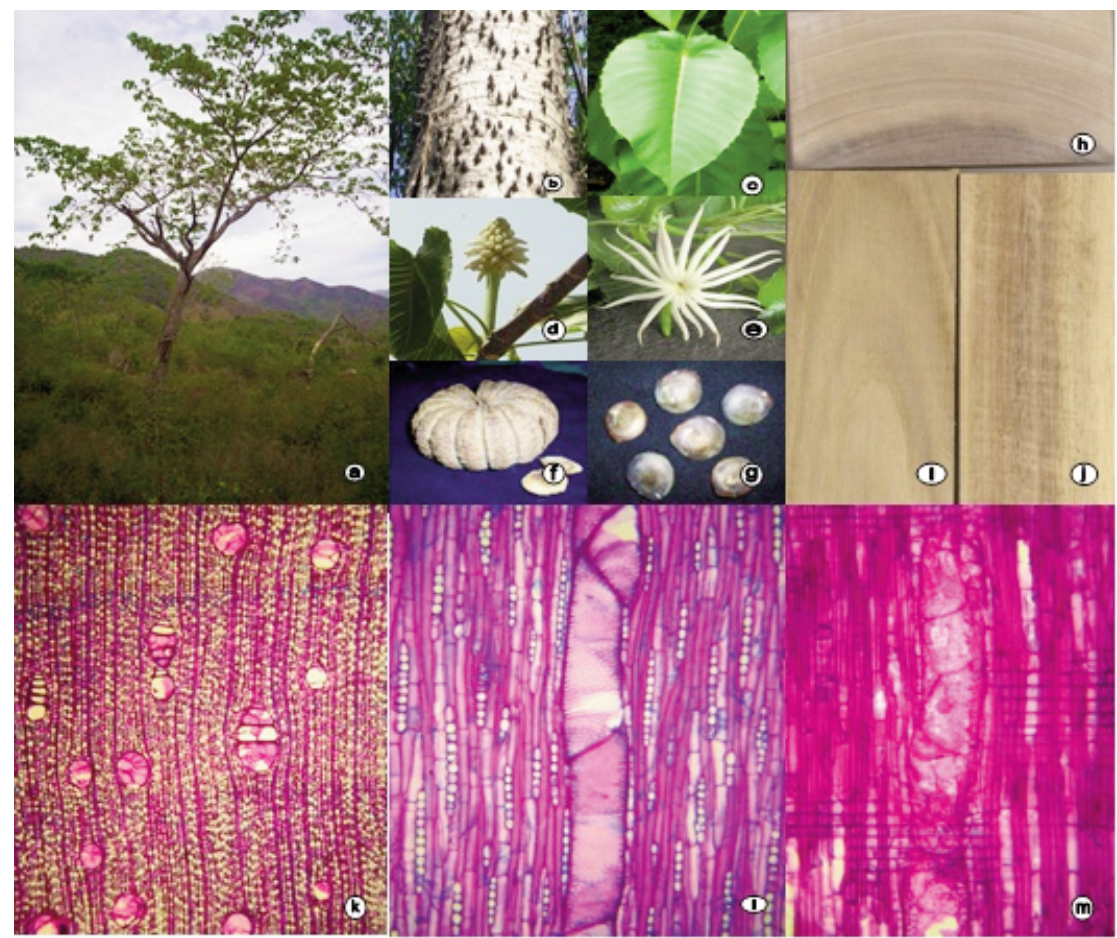

Lámina 2. Hura polyandra. a. árbol. b. corteza. c. hoja. d. flor masculina. e. flor femenina. $f$. fruto. $g$. semillas. Tablillas: $h$. transversal. $i$. tangencial. $j$. radial.

Cortes: $k$. transversal (5x). I. tangencial (10x). m. radial (10x). 
Microscópicas (Lámina 2, figs. k-m; Lámina 3, figs. d-f; Tabla 2)

La madera es de porosidad difusa, los poros son pocos, de diámetro tangencial mediano, la mayoría múltiples de 4 a 6 , algunos solitarios. Los elementos vasculares son medianos, sus paredes con puntuaciones areoladas alternas, ovales y platina de perforación simple. Algunos presentan tílides. El parénquima axial es apotraqueal en bandas, de una hilera de células de ancho. Algunas células parenquimáticas presentan cristales prismáticos dentro de cámaras. Los radios son homogéneos, uniseriados, moderadamente numerosos, extremadamente bajos y muy finos. Las fibras son de longitud mediana, diámetro fino y pared gruesa. Los anillos de crecimiento miden de $2 \mathrm{~mm}$ a $15 \mathrm{~mm}$ de ancho, marcados por 3 a 4 hileras de fibras.

\section{Características físicas (Tabla 3)}

La madera es moderadamente pesada, de contracciones bajas, muy estable y salida de agua libre lenta.

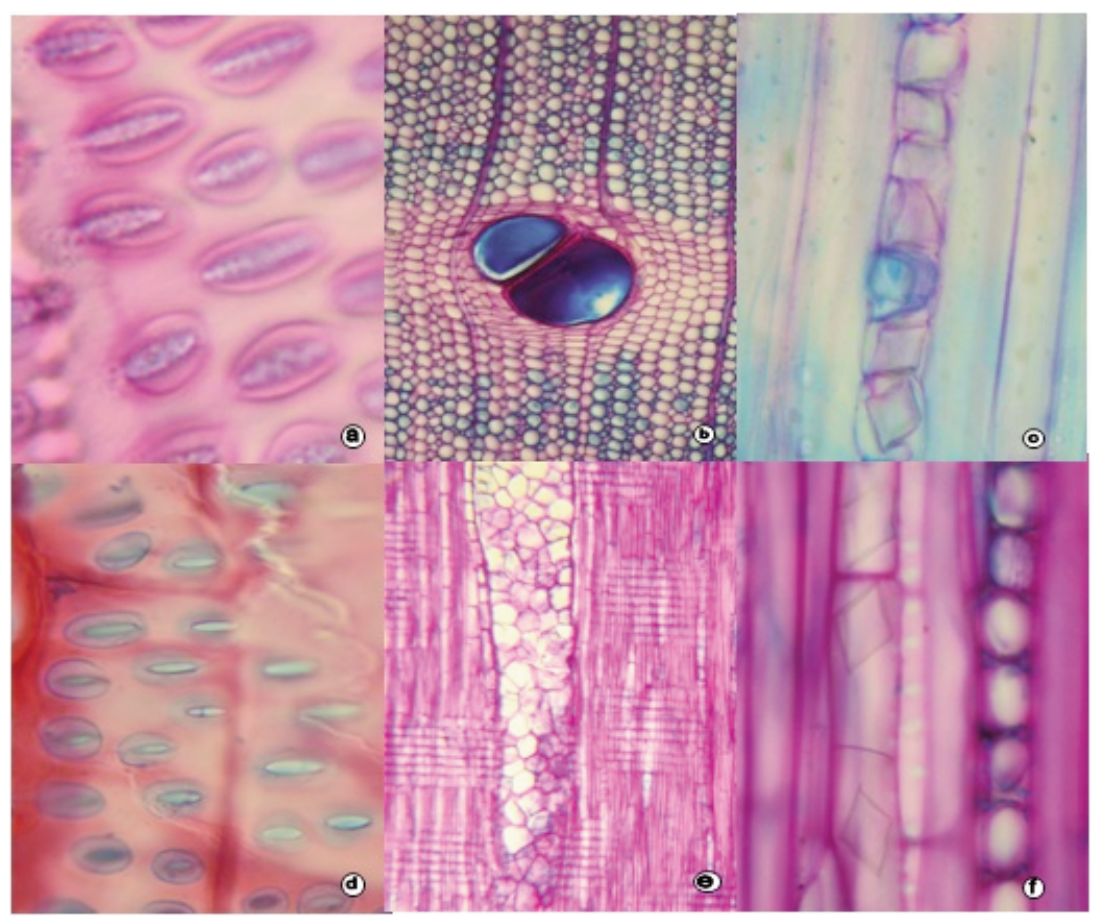

Lámina 3. Puntuaciones y contenidos celulares de las especies. a-c. Enterolobium cyclocarpum. a. Puntuaciones areoladas ornamentadas en vasos (100x). b. Gomas en vasos (20x). c. Cristales prismáticos en parénquima (40x). $d$-f. Hura polyandra. d. Puntuaciones areoladas no ornamentadas en vasos (100x). e. Tílides (10x). $f$. Cristales prismáticos en parénquima (40x). 


\section{Características mecánicas (Tabla 4)}

La madera es muy dura, rígida, resistente a la compresión, muy resistente al cortante paralelo y de tenacidad media.

\section{Características de secado (Tabla 6)}

La curva de secado correspondiente a esta especie se muestra en la figura 4.

La madera es de secado lento (tiempo de secado 35 días para alcanzar el $\mathrm{CHE}$ ).

\section{DISCUSIÓN}

La madera de las dos especies comparte características en cuanto a la diferencia en tonalidad entre albura y duramen; la albura es blanca y el duramen es castaño claro en ambas, no tienen olor ni sabor característicos, su brillo es mediano, el veteado suave y la textura fina. En Enterolobium cyclocarpum el hilo es oblicuo, los anillos están marcados por 2 a 4 hileras de fibras y en Hura polyandra el hilo es recto y los anillos están marcados por 2 a 3 hileras de fibras.

En las características microscópicas coinciden en la porosidad difusa, con vasos de diámetro mediano, con puntuaciones areoladas alternas ovales y platina de perforación simple, el parénquima axial con cristales prismáticos, los radios son uniseriados, homogéneos, extremadamente bajos y las fibras de longitud mediana y diámetro fino.

Por otro lado presentan diferencias en su anatomía. En E. cyclocarpum los poros son muy pocos, la mayoría solitarios y unos cuantos múltiples. Los elementos vasculares son muy cortos, con puntuaciones ornamentadas, algunos con gomas. El parénquima axial es paratraqueal vasicéntrico. Los radios pocos, extremadamente finos. Las fibras de pared delgada. Los anillos de crecimiento de $10 \mathrm{~mm}$ a $25 \mathrm{~mm}$ de ancho.

En $H$. polyandra los poros son pocos, la mayoría múltiples y algunos solitarios. Los elementos vasculares son medianos, con puntuaciones no ornamentadas, algunos con tílides. El parénquima es axial apotraqueal en bandas, de una hilera de células de ancho. Los radios son moderadamente numerosos, muy finos. Las fibras tienen pared gruesa. Los anillos de crecimiento son de $2 \mathrm{~mm}$ a $15 \mathrm{~mm}$ de ancho.

En las propiedades físicas, mecánicas y tiempo de secado, únicamente coinciden en las contracciones tangenciales que son bajas y en las demás propiedades son diferentes. E. cyclocarpum es una madera muy liviana, de contracción tangencial baja y contracciones radial y volumétrica muy bajas, poco estable, con punto de saturación de la fibra medio. $H$. polyandra es una madera moderadamente pesada, cuyas contracciones son bajas, estable dimensionalmente y punto de saturación menor que E. cyclocarpum. Es suave, flexible, poco resistente a la compresión, moderadamente resistente al cortante y poco resistente al impacto. El tiempo de secado es diferente para cada una.

Estas diferencias de comportamiento se explican en gran medida por tratarse de especies con densidades diferentes, que redundan en repuesta a acciones mecánicas diferentes: mayores valores para la madera más densa (Dávalos y Bárcenas, 1999). La misma respuesta se presenta, en general, para las propiedades físicas: las contracciones son mayores para la madera más densa, conforme a la teoría que indica que las contracciones son proporcionales a la densidad (Panshin y de Zeew, 1970) y en consecuencia al grosor de la pared 
celular. En cuanto a su comparación con especies parecidas de otras regiones 0 países, se encontró que Hura crepitans, especie sudamericana con densidad básica promedio de 0,42 tiene una razón Contracción tangencial/Contracción radial (CT/CT) promedio (coeficiente de anisotropía) de 1,6 (Keenan y Tejada, 1987), comparable a la obtenida en este estudio para $H$. polyandra $(1,65)$. EcheniqueManrique (1970) señala que los valores de $H$. crepitans pueden compararse directamente con $H$. polyandra porque es difícil distinguir entre la madera de ambas especies, salvo por ciertos caracteres botánicos. En el caso de E. cyclocarpum, Robles (1978) determinó un coeficiente de anisotropía de 2,71, comparable con el registrado aquí de 2,52. Estos datos son muy parecidos a valores obtenidos en especies tropicales de Puerto Rico de densidad similar a E. cyclocarpum (promedio 0,28 ) para las que se calcularon valores de CT/CR promedio de 2,27 (Longwood, 1961).

E. cyclocarpum coincide en la diferencia entre albura y duramen con anteriores trabajos (Cárdenas, 1971; Corral, 1985; Silva, 2008), es diferente en el veteado, así como en la textura, hilo y la presencia de anillos de crecimiento. Estas diferencias son debidas al tamaño de los elementos celulares como el diámetro de los poros, la altura corta, la anchura fina de los radios que producen una textura fina. Por otro lado, el hilo es oblicuo, de manera semejante a lo comúnmente encontrado en especies tropicales (Silva, 2008).

En lo que respecta a las propiedades físicas, es similar a la densidad de ejemplares de las selvas de Campeche $(0,29)$ (Robles, 1978) y menor a algunas de Yucatán $(0,37)$ (Gonzalez et al., 2004). La densidad de $H$. polyandra $(0,48)$ fue similar a lo mencionado por EcheniqueManrique (1970) $(0,36-0,44)$ y también es semejante a los datos del trabajo de Silva (2008) $(0,47-0,68)$. Tales valores están en función de las dimensiones de sus elementos celulares y se explican principalmente por el grosor de la pared de sus fibras (León, 2010). Silva (2008) presenta valores similares $(0,47)$ para sus ejemplares, clasificándola como una madera de peso mediano, contracciones bajas y buena estabilidad dimensional, lo que coincide con este estudio.

Las bajas contracciones de ambas especies se pueden deber a que haya un menor número de radicales libres en las paredes celulares, para albergar moléculas de agua; la especie con menor valor de coeficiente de anisotropía, $H$. polyandra, tiene mayor estabilidad dimensional y por lo tanto, menor riesgo de que aparezcan grietas de la madera durante el proceso de secado, que a su vez es más lento que el de la especie más ligera. Otras variables que influyen en este comportamiento son las dimensiones de los elementos constitutivos y los tipos de contenidos presentes que retrasan la salida del agua libre (De la Paz y Dávalos, 2008).

En el presente estudio, los valores del módulo de ruptura en la prueba de flexión (41,4 MPa) y los valores de dureza (2,28 kN y3,04 kN para lateral y longitudinal, respectivamente) de E. cyclocarpum, fueron inferiores a lo que presenta Silva (2008), (42,2 MPa a 58,8 MPa) para una densidad de 0,38 - 0,52, asimismo, se encontró un valor de cortante paralelo $(7,1 \mathrm{MPa})$ igual a lo determinado por González et al. (2004) $(7,2 \mathrm{MPa})$ con madera de mayor densidad $(0,37)$. La resistencia al impacto está en relación directa al grosor de la pared de las fibras. Las propiedades mecánicas de $H$. polyandra la describen como una madera dura, resistente a la flexión, a la compresión perpendicular y paralela, así como al cortante paralelo. 
Estos resultados tienen valores más altosque los obtenidos por EcheniqueManrique (1970) y menores a lo descrito por Silva (2008) en madera con menor y similar densidad, respectivamente.

En ambas especies se aprecian características que dificultan su aserrío. El aserrín de E. cyclocarpum afecta las vías respiratorias de algunas personas y la savia de $H$. polyandra es urticante para la piel y los ojos.

De acuerdo con una estimación previa del crecimiento se encontró que la edad de los árboles estudiados de $E$. cyclocarpum es de entre 12 y 18 años y los de $H$. polyandra de aproximadamente 20 años (Pineda et al., 2011).

Los resultados obtenidos en este estudio mostraron que la madera de $E$. cyclocarpum es apta para usarse en artesanías, decoración de interiores, chapa, lambrín y muebles, quedando descartados aquellos usos que requieran de piezas con resistencia a esfuerzos estructurales altos como construcción o piezas sometidas a alto impacto como mangos de herramientas $\mathrm{o}$ al desgaste como duelas. En ocasiones se emplea en la forma de utensilios de cocina, lo que concuerda con las características organolépticas determinadas en este trabajo. $H$. polyandra puede emplearse en construcciones rurales, chapas, embalajes, muebles y madera contrachapada (triplay). No se recomienda para piezas de alta resistencia al impacto como mangos de herramientas.

\section{CONCLUSIONES}

El conocimiento de las características de la madera de Enterolobium cyclocarpum (parota) y Hura polyandra (habillo), aunado a información sobre su crecimiento y fenología, permitirá a los produc- tores, industriales y artesanos emplearla de manera más eficiente por tener características muy adecuadas para usos prácticos amplios. E. cyclocarpum es apta para múltiples productos por ser una madera liviana, más no para uso estructural debido a la baja resistencia que presenta ante los diferentes esfuerzos. $H$. polyandra se podría utilizar en una gama de aplicaciones que hasta el momento no se le han dado en la región. Dado el valor potencial de ambas especies, se recomienda que los propietarios del recurso consideren emprender acciones de certificación del manejo forestal por parte de entidades reconocidas nacionalmente.

\section{AGRADECIMIENTOS}

A los señores Hipólito Herrera y Félix Bustos por las facilidades para la recolección del material de estudio. A la M. en I. Reyna Paula Zárate Morales del Inecol, por la preparación del material para las pruebas y la realización de las pruebas físicas y mecánicas, a la doctora Guadalupe M. Bárcenas del Inecol por las facilidades prestadas para el uso del LINCELaboratorio de Pruebas, a la doctora Alejandra Quintanar de la UAMI por su ayuda en la anatomía de las puntuaciones, a la Coordinación del Doctorado en Ciencias Biológicas y de la Salud por el apoyo económico y al Conacyt por la beca de posgrado.

\section{REFERENCIAS}

ASTM (American Society for Testing and Materials). 2010. ASTM D143-09. Standard methods of testing small clear specimens of timber. ASTM International, West Conshohocken, PA. Vol. 4.10. 31 p. 
ASTM. (American Society for Testing and Materials). 1998. ASTM D2915. Standard methods for evaluating allowable properties for grades of structural lumber. Annual Book of Standards. 1970 to 2000 edition. West Conshohocken, PA.

Chattaway, M. 1932. Proposed standards for numerical values used in describing woods. Tropical Woods 9:20-28.

Chattaway, M. 1955. Crystals in woody tissues I. Tropical Woods 102:55-74.

Cárdenas, E. 1971. Estudio anatómico de la madera de 8 especies de leguminosas. Tesis. Escuela Nacional de Ciencias Biológicas. IPN. 54p.

Corral, G. 1985. Características anatómicas de la madera de once especies tropicales. Bol. Téc. Inst. Nac. Invest. For. México, núm. 127.

Dávalos, R. y G. Bárcenas. 1999. Clasificación de las propiedades mecánicas de las maderas mexicanas en condición seca. Madera y Bosques 5(1):61-69.

De la Paz Pérez, C. y R. Dávalos-Sotelo. 2008. Algunas características anatómicas y tecnológicas de 24 especies de Quercus (encinos) de México. Madera y Bosques 14(3):43-80.

Echenique-Manrique, R. 1970. Descripción, características y usos de 25 maderas tropicales mexicanas. Maderas de México. Número 1. Cámara Nacional de la Industria de la Construcción, México. 237p.

Echenique-Manrique, R., J. Barajas, L. Pinzón y V. Pérez. 1975. Características tecnológicas de la madera de 10 especies en: Estudio botánico y ecológico de la región del río Uxpanapa, Veracruz núm. 1. Inireb. 66 p.

González I, M. Cerón y L. Solís. 2004. Esfuerzos cortante y de tensión paralelos a la fibra en madera tropical de crecimiento rápido. Ingeniería 8(1):23-32.

Guridi G., L. 1980. La madera en las artesanías del Estado de Michoacán. Bol. Div. Inst. Nac. Invest. For. México núm. 50.

IAWA Committee. 1937. Standards terms of length of vessels members and wood fibers. Tropical Woods 51:21-22.

IAWA Committee. 1939. Standards terms of size for vessels diameter and ray width. Tropical Woods 59:51-52.

IAWA Committee. 1964. Multilingual glossary of terms used in wood anatomy. Committee on Nomenclature. Suiza. $186 p$.

IAWA Committee.1989. IAWA list of microscopic features for hardwood identification. IAWA Bulletin 10(3):219-332.

INEGI. 2005. Conjunto de datos vectoriales de la carta de uso del suelo y vegetación: escala 1:250 000. Serie III (continuo nacional). Instituto Nacional de Estadística, Geografía e Informática, Aguascalientes, Ags.

INEGI. 2009. Prontuario de información geográfica municipal de los Estados Unidos Mexicanos. http://www.inegi. org.mx/sistemas/mexicocifras/datosgeograficos/12/12038.pdf. Consultado el 12 de enero de 2012.

Johansen, D. 1940. Plant microtechnique. McGraw-Hill. Nueva York. 523p. 
Keenan, F.J. y M. Tejada. 1987. Maderas tropicales como material de construcción en los países del Grupo Andino de América del Sur. Centro Internacional de Investigaciones para el Desarrollo, Bogotá, Colombia. 147 p.

León, W. 2010. Anatomía y densidad o peso específico de la madera. Rev. For. Venez. 54:67-76.

Longwood, F.R. 1961. Puerto Rican woods. Their machining, seasoning and related characteristics. Agriculture Handbook num. 205, USDAFS, Washington, D.C. 98p.

Morán, M.M., F. Carrera G., J.J. Campos A., L. Bastiaan, D. Delgado y G. Galloway. 2008. Evaluación de la sostenibilidad del manejo forestal comunitario en ejidos del estado de Guerrero, en: http://web.catie.ac .cr/informacion/RFCA/rev4950/Pages\%20124-130.pdf. Consultado el 25 de enero de 2012.

Munsell. 1990. Munsell soil color charts. Baltimore, Maryland. 17p.

Panshin, A.J. y C. de Zeew. 1970. Textbook of wood technology. Vol. 1, McGraw-Hill Book Co., Nueva York. $705 p$.

Pennington, T.D. y J. Sarukhán. 2005. Árboles tropicales de México: Manual para la identificación de las principales especies. UNAM-FCE México. D.F. 521p.

Pineda, E., C. de la Paz Pérez, R. Dávalos, J.I. Valdez y R.P. Zárate. 2011. Crecimiento, anatomía y propiedades físico-mecánicas de la madera de dos especies tropicales de Costa Grande, Guerrero. Memo- rias del XVI Foro de estudios sobre Guerrero. p 160-163.

Pineda, E., C. de la Paz Pérez y J.I. Valdez. 2012. El aprovechamiento maderable en Costa Grande, Guerrero. Biodiversitas 102:6-12.

Robles, F. 1978. Propiedades y usos de 14 especies tropicales de rápido crecimiento del campo experimental forestal El Tormento. Bol. Inst. Nac. Invest. For. México núm. 16.

Rodríguez, A. 2009. Caracterización de la fabricación de muebles de madera en el municipio de La Unión del estado de Guerrero. Tesis profesional. División de Ciencias Forestales, Universidad Autónoma Chapingo, México.

Román, L., A. Mora y A. Gallegos. 2011. Árboles tropicales de uso múltiple en la costa de Jalisco, México. In: Bosques y arboles del trópico mexicano: estructura, crecimiento y usos. J.I. Valdez, A. Mora y R. Endara (eds.). Prometeo Eds. 107 p.

Sallenave, P. 1955. Proprietes physiques et mechaniques des bois tropicaux de Lunion Francaise. Centro Techique Forestier Tropical. Nagent. Sur-Marne.

Sandoval, E. 2005. Técnicas aplicadas al estudio de la anatomía vegetal. Cuadernos del Instituto de Biología 38. UNAM. México, D.F. 281p.

Silva, J.A. 2008. Fichas técnicas sobre características tecnológicas y usos de maderas comercializadas en México. Coordinación, Educación y Desarrollo Tecnológico. Conafor. $62 p$. 
SMN (Servicio Meteorológico Nacional). 2012. Datos de precipitación y temperatura de la estación automática Vallecitos de Zaragoza. Mayo de 2012.
Tortorelli, L. 1956. Maderas y bosques argentinos. ACME. Buenos Aires. 910p.

Zavala, D. 2000. Secado de maderas en estufas convencionales. Madera $y$ Bosques 6(1):41-54. 18(3):53-71. 